\title{
Significados da qualidade de vida para pacientes com melasma facial
}

Meanings of quality of life for patients with facial melasma

Significados de la calidad de vida para pacientes con melasma facial

Camila Fernandes Pollo', Luciane Donida Bartoli Miot², Hélio Amante Miot², Silmara Meneguin ${ }^{1}$

ORCID IDs

Pollo CF (iD https://orcid.org/0000-0003-0264-5841

Miot LDB (iD https://orcid.org/0000-0002-2388-7842

Miot HA (D) https://orcid.org/0000-0002-2596-9294

Meneguin S (iD https://orcid.org/0000-0003-3853-5134

\section{COMO CITAR}

Pollo CF, Miot LDB, Miot HA, Meneguin S. Significados da qualidade de vida para pacientes com melasma facial. ESTIMA, Braz.J. Enterostomal Ther., 16: e3318. https://doi.org/10.30886/estima.v16.626 PT.

\section{RESUMO}

Objetivo: Desvelar os significados atribuídos à qualidade de vida por pacientes com melasma facial. Métodos: Pesquisa qualitativa utilizando a técnica de grupo focal, com 10 pessoas com melasma facial oriundas de hospital público e clínica privada de município no interior do estado de São Paulo. O referencial metodológico utilizado foi análise de conteúdo. Resultados: Percebe-se, na fala dos entrevistados, o incômodo gerado pela opinião de outros, com perguntas curiosas e até maldosas relacionadas à mancha. Há dificuldade para esconder as lesões e a aparência de descaso com a pele também é motivo de angústia para as participantes. Conclusão: Por acometer principalmente a face, o que o torna facilmente visível, o melasma incomoda e tem impacto negativo na qualidade de vida, pois afeta o bem-estar psicológico e emocional dos pacientes.

DESCRITORES: Qualidade de vida; Melasma; Hiperpigmentação; Enfermagem.

\footnotetext{
"Universidade Estadual Paulista "Júlio de Mesquita Filho" - Faculdade de Medicina de Botucatu - Departamento de Enfermagem Botucatu/SP - Brasil.

2Universidade Estadual Paulista "Júlio de Mesquita Filho" - Faculdade de Medicina de Botucatu - Departamento de Dermatologia e Radioterapia - Botucatu/SP - Brasil.

Autor correspondente: Silmara Meneguin | Universidade Estadual Paulista "Júlio de Mesquita Filho" - Faculdade de Medicina de Botucatu - Departamento de Enfermagem - Av. Professor Montenegro, s/n | CEP: 18618-000 - Botucatu/SP - Brasil | E-mail: silmeneguin@fmb.unesp.br

Recebido: Jul. 07, 2018| Aceito: Out. 10, 2018
} 


\begin{abstract}
Objective: To exhibit the meanings attributed to quality of life by patients with facial melasma. Methods: Qualitative research using the focal group technique, with 10 people with facial melasma from a public hospital and private clinic of a municipality in the interior of the state of São Paulo. The methodological indicative used was content analysis. Results: In the interviewees' speech, one perceives the annoyance generated by the opinion of others, with curious and even malicious questions related to the blemish. There is difficulty to hide the injuries and the appearance of skin neglect is also cause for distress for the participants. Conclusion: Because it affects the face, which makes it easily visible, melasma is uncomfortable and has a negative impact on the quality of life, since it affects patients' psychological and emotional well-being.
\end{abstract}

DESCRIPTORS: Quality of life; Melasma; Hyperpigmentation; Nursing.

\title{
RESUMEN
}

Objetivo: Desvelar los significados atribuidos a la calidad de vida por pacientes con melasma (también conocido por cloasma) facial. Métodos: Investigación cualitativa utilizando la técnica de grupo focal, con 10 personas con melasma facial oriundas de hospital público y clínica privada de municipio en el interior del estado de São Paulo. El referencial metodológico utilizado fue el análisis de contenido. Resultados: Se percibe, en el habla de los entrevistados, el incómodo generado por la opinión de otros, con preguntas curiosas e incluso maldosas relacionadas con la mancha. Hay dificultad para ocultar las lesiones y la apariencia de la negligencia con la piel también es motivo de angustia para las participantes. Conclusión: Por acometer principalmente la cara, lo que lo hace fácilmente visible, el melasma incomoda y tiene impacto negativo en la calidad de vida, pues afecta el bienestar psicológico y emocional de los pacientes.

DESCRIPTORES: Calidad de vida; Melasma; Hiperpigmentación; Enfermería.

\section{INTRODUÇÃO}

Melasma é uma alteração pigmentar comum, crônica e recorrente que se caracteriza por máculas hiperpigmentadas assintomáticas e simétricas na pele, principalmente na face. Ocorre devido à hipermelanogênese local e atinge milhões de pessoas em todo o mundo ${ }^{1}$. Geralmente ocorre em um de três padrões clínicos. O mais comum é o centro facial, enquanto os tipos menos comuns são o malar e o mandibular².

Mulheres em idade fértil são as mais comprometidas e as áreas fotoexpostas são as mais acometidas, principalmente a face ${ }^{3}$. Melasma acomete todas as etnias e populações, porém estudos epidemiológicos referem maior predominância entre fenótipos mais pigmentados, como asiáticos do Leste (japoneses, coreanos e chineses), indianos, paquistaneses, povos do Oriente Médio e África mediterrânea. Nas Américas, é comum entre hispânicos e brasileiros que habitam regiões intertropicais, onde há maior exposição à radiação ultravioleta ${ }^{1,4}$.

A predisposição genética pode ser notada com a alta incidência de história familiar de melasma entre pacientes com a doença. Em estudo brasileiro com 302 mulheres portadoras de melasma facial, houve referência de 47,6\% de familiares de primeiro grau acometidos ${ }^{5}$.
Melasma é uma doença geralmente de difícil tratamento, de evolução longa e com frequentes exacerbações que podem influenciar na percepção da qualidade de vida pelos pacientes ${ }^{6}$.

Qualidade de vida $(\mathrm{QV})$ é definida pela Organização Mundial da Saúde (OMS) como a percepção do indivíduo de sua posição na vida, no contexto da cultura e sistemas de valores nos quais ele vive em relação a seus objetivos, expectativas, padrões e preocupações ${ }^{7}$.

No âmbito da clínica, está relacionada à repercussão ou ao tratamento de doenças que podem influenciar na percepção do conceito. Nesse sentido, o impacto da doença na $Q V$ dos pacientes está sendo, cada vez mais, enfatizado em ensaios clínicos terapêuticos como um desfecho relevante ${ }^{8}$.

No paciente clínico, as dermatoses podem afetar a autoimagem e têm grande potencial para desencadear processos que afetam a autoestima, contribuindo para provocar sentimentos que podem manifestar-se como ansiedade, tristeza ou até mesmo quadros depressivos. Sabe-se que a intensidade do prejuízo na QV é proporcional ao tempo que a pessoa acometida convive com o melasma9 .

No entanto, tem-se dado pouca ênfase à percepção subjetiva do indivíduo com melasma em relação à sua $\mathrm{QV}$. 
A maioria dos estudos realiza avaliação do constructo por meio de instrumentos psicométricos ${ }^{10}$.

Assim sendo, esta investigação pretende valorizar a singularidade do indivíduo com melasma e, dessa forma, contribuir para a construção do conhecimento na área de enfermagem em dermatologia, a partir das vivências de pacientes dermatológicos.

\section{OBJETIVO}

Desvelar os significados atribuídos à $\mathrm{QV}$ por pacientes com melasma facial.

\section{MÉTODOS}

Estudo descritivo, exploratório, com abordagem qualitativa, realizado em hospital público universitário no interior do estado de São Paulo e em clínica privada do mesmo município em março de 2014.

Estabeleceram-se, como critérios de inclusão, pacientes portadores de melasma em tratamento clínico e maiores de 18 anos.

Após essa etapa, foram sorteados aleatoriamente cinco pacientes da lista de atendidos em cada um dos locais de pesquisa e então realizou-se o convite para participar do grupo focal. Optou-se por esse método por ser uma técnica utilizada para obtenção de dados a partir de discussões previamente planejadas em que os participantes expressam suas experiências, valores, crenças e atitudes sobre questões específicas ${ }^{11}$.

Nesse método, a discussão deve ser conduzida por um moderador por aproximadamente 2 horas em local privativo $^{11}$. O registro da discussão é realizado por um observador que se encarrega de monitorar o equipamento de gravação, de forma ágil e discreta, visando não deslocar a atenção do grupo para seus movimentos. Também faz um breve registro em cada fala dos participantes, para facilitar a posterior transcrição ${ }^{12}$.

Dados sociodemográficos foram coletados por meio de instrumento desenvolvido para o estudo e obtido dos registros de prontuário. $\mathrm{Na}$ sequência, realizou-se o grupo focal, tendo como questões norteadoras: 1) Na opinião de vocês, o melasma interfere na $\mathrm{QV}$ ?; e 2) Em quais aspectos da $Q V$ vocês acham que o melasma interfere?
As falas foram gravadas e posteriormente transcritas utilizando-se a análise de conteúdo de Bardin ${ }^{13}$. Nessa técnica, após a transcrição literal, realiza-se a pré-análise, fase de organização do conteúdo que tem por finalidade sistematizar as ideias para, na sequência, proceder com a exploração do material que envolve essencialmente a operação de categorização. Por fim, num terceiro momento, ocorre a análise e interpretação dos dados.

\section{RESULTADOS}

Participaram do estudo 10 pacientes, todas do sexo feminino, com idade média de 47,3 anos. Dessas, cinco são oriundas de clínica privada e a outra metade do hospital público. Quanto à escolaridade, cinco têm nível médio, quatro nível superior e somente uma nível fundamental.

Para melhor compreensão dos resultados, as unidades de significado foram agrupadas em três temas: aspectos físicos/aparência, tratamento clínico e aspectos sociais/ emocionais com suas respectivas categorias. Os participantes (P) estão identificados no texto com a numeração que lhes foi atribuída na transcrição do grupo focal.

Do primeiro tema, aspectos físicos/aparência, emergiram as categorias: indagações curiosas e maldosas a respeito da mancha, dificuldade para esconder a lesão e aparência de pele não cuidada.

Percebe-se, na fala dos entrevistados, o incômodo gerado pela opinião de outros, com perguntas curiosas e até maldosas relacionadas à mancha, pois o aspecto visível das lesões de pele e seu impacto psicológico interferem na QV dos pacientes ${ }^{14}$.

Eu tive a infelicidade de uma pessoa falar: "você não tira o buço?”. (P1)

Viu, você nunca foi no dermatologista! (P2)

O maior constrangimento é a pessoa perguntar para você: "viu, e essa mancha, você não faz nada pra isso?". (P3)

Dificuldade para esconder a mancha e aparência de descaso com a pele também geram angústia, pois as participantes relatam que é muito difícil encontrar um produto que cubra completamente a mancha, o que acaba gerando um aspecto de pele descuidada. 
Alguns autores descrevem que lesões de pele na face são mais perceptíveis para o olhar do outro. Portanto, a aparência visual terá papel fundamental na percepção de si mesmo ${ }^{15}$.

Já fui fazer maquiagem e a pessoa virar e falar: "não tem jeito, não tem como esconder”. (P2)

Eu vou passar outra coisa no rosto e acentua assim mil vezes mais a mancha, você não consegue esconder, né? (P5)

No segundo tema, foram mencionados os tratamentos clínicos pelas participantes, dos quais emergiram as seguintes categorias: incômodo com o filtro solar, com o próprio tratamento para clarear as manchas e com o custo do tratamento.

O cuidado com a doença e seu tratamento também representam dificuldades que podem prejudicar o lazer e as atividades de trabalho ${ }^{16}$.

As participantes relataram insatisfação em relação aos protetores solares disponíveis no mercado, devido ao fato de não terem boa cobertura; devido à textura do produto não permitir absorção adequada, deixando a pele com aspecto oleoso e lambuzado; e, por fim, por não serem adequados à pele da mulher brasileira, haja vista que a grande maioria desses produtos são importados.

Os filtros solares, eles são para as mulheres europeias, americanas, não tem para a mulher brasileira, não é? Então, tudo mela, tudo gruda, sabe? Você está bem arrumada, mas você está melando, então você vai cumprimentar uma pessoa, você se sente mal por causa daquela grudação. (P3)

Com base, se você for dar um beijo na pessoa, se a pessoa for mais clara que a gente, fica aquela marca na bochecha da pessoa. $(\mathrm{P} 2)$

O telefone de casa, o meu marido fala que está tudo branco, ele reclama de ter que limpar, eu acabo sujando o tempo todo. (P6)

O próprio tratamento para clareamento das manchas também gera desconforto e impacta na QV das participantes. Algumas pacientes relataram não aderir ao tratamento no verão, pois é tão forte que, além de deixarem a pele vermelha, ardida e haver maior risco de escurecer novamente a mancha, os cremes utilizados no tratamento sujam e até danificam a roupa de cama.

Eu não faço tratamento no verão. Eu ando a pé, não tenho carro, e eu tenho medo de ficar mais manchada se eu continuar o tratamento no verão. (P3)

A minha piorou com o tratamento no verão. Aliás, meu marido me cobra: "mas você fez errado!". (P6)

O cheiro me incomoda, você vai dar um beijinho no marido, você está toda fedida. (P7)

É forte, ele chega a corroer a fronha, e a mancha ele não tira. (P4)

Eu tive uma alergia enorme pela hidroquinona. (P5)

Tratamento de pele é bem caro. (P3)

Eu acho bem caro. (P2)

Protetor solar mesmo, se você for ver quanto você gasta por mês... (P6)

No terceiro tema, desvelaram-se os aspectos sociais e emocionais com suas respectivas categorias: incômodo com o olhar velado do outro, com fotografias e a própria imagem ao se olhar no espelho.

Em uma sociedade que valoriza o belo e tem padrões estéticos preestabelecidos, a pele pode ser considerada um cartão de visitas que atrai, assim, os olhares críticos e exigentes de indivíduos. Quando íntegra e saudável, a pele promove a relação entre as pessoas e facilita seu desenvolvimento nos aspectos sociais, emocionais e sexuais. Porém, quando acometida por afecções cutâneas, pode trazer sérias consequências ${ }^{17}$.

A impressão é que as pessoas estão conversando com a gente e olhando direto para aquilo, mesmo que não percebam. (P2)

A gente tem a sensação de que as pessoas estão olhando para a mancha, e não para a gente, e não no olho da gente. (P4)

$\mathrm{Na}$ fotografia fica bem escura. (P3) 
Quando a gente olha no espelho, a gente só vê a mancha, o restante nada mais chama sua atenção. (P5)

A opinião dos outros não me incomoda muito, não. Acho que é mais quando eu olho no espelho mesmo. (P3)

\section{DISCUSSÃO}

Os resultados indicam que o melasma é uma dermatose frequente e, por acometer áreas expostas, produzindo o aspecto de descuido e ser de difícil camuflagem, maximiza seu impacto na imagem corporal e nas relações interpessoais. Estudos mostram que pacientes acometidos por essa doença sentem-se aborrecidos, menos atraentes e utilizam cosméticos para cobrir as manchas ${ }^{18}$.

$\mathrm{O}$ paciente acredita que as pessoas focalizam sua pele em vez de prestar atenção no que ele está dizendo, o que compromete as atividades sociais e de lazer. Assim, é possível afirmar que melasma gera grande impacto na QV desses pacientes, por se tratar de dermatose crônica e, por vezes, estigmatizante $^{19,20}$.

Apesar de grande parte dos pacientes dermatológicos não apresentarem doenças com risco de morte, as dermatoses originam, frequentemente, grande impacto no estado emocional, nas relações sociais e nas atividades cotidianas dos doentes, pelos estigmas causados por manifestações muito aparentes ${ }^{21}$.

Doenças de pele prejudicam a autoimagem e têm potencial para conduzir a depressão e a ansiedade tanto quanto doenças sistêmicas graves. Apesar de não serem ameaçadoras à vida, nem fisicamente debilitantes, podem afetar gravemente funções psicológicas e sociais dos indivíduos ${ }^{22}$.

Nesse sentido, à medida que uma pessoa percebe mudanças físicas em sua aparência, essa condição pode causar sofrimento e alterações em sua vida, implicando prejuízos na rotina diária, em socialização e perdas de forma geral. Dados sociológicos e psicológicos cada vez mais indicam que problemas de pele podem ter sérias consequências, pois geram vergonha, ansiedade e constrangimento social ${ }^{23}$.

A banalização do problema pode ser entendida à luz da formação educacional tradicional em saúde: o modelo biomédico. Fundado no pensamento simplificador e reducionista, leva o estudante a uma visão fragmentada da complexidade da vida ${ }^{24}$. Estudos apontam o entrave da equipe em transcender os limites traçados para sua atuação ${ }^{25}$.

Um estudo evidencia a necessidade de processos de educação dirigidos às equipes de saúde com foco na valorização do melasma, considerando que se trata de problema de baixa morbidade, mas que impacta na QV das mulheres ${ }^{25}$.

$\mathrm{O}$ melasma causa impacto negativo na $\mathrm{QV}$, principalmente por acometer a face e a imagem corporal. Devido à insatisfação com a aparência, as pacientes apresentam comprometimento da autoestima, com repercussões na vida pessoal e profissional ${ }^{23}$.

Estudo recente com abordagem qualitativa aponta dados que corroboram com os achados desta investigação ${ }^{26}$.

Por serem aparentes, as dermatoses repercutem facilmente na vida pessoal, profissional e social de seus portadores. Em vista disso, faz-se necessária a valorização do tema visando à avaliação global desses indivíduos.

A carência de estudos que avaliam a percepção da QV sob a ótica dos pacientes apresentou-se como limitação para comparar os achados deste estudo.

\section{CONCLUSÃO}

Por acometer principalmente a face, o que o torna facilmente visível, o melasma incomoda e tem impacto negativo na $\mathrm{QV}$, pois afeta o bem-estar psicológico e emocional dos pacientes.

Discursos apontam que QV é uma concepção subjetiva, atrelada a valores e influenciada pelas repercussões do processo saúde-doença em todas as suas dimensões. Esse dado remete à necessidade de não considerar essa afecção somente um problema estético.

Nesse contexto, a enfermagem pode trazer importante contribuição com sua atuação na atenção integral à saúde, ampliando seu campo de visão e dando sentido às questões do outro como uma pessoa única, com suas próprias necessidades e expectativas.

\section{CONTRIBUIÇÃO DOS AUTORES}

Conceitualização: Pollo CF; Meneguin S e Miot HA; Metodologia, Pollo CF; Meneguin S e Miot HA; Investigação: Pollo CF; Redação - Primeira versão, Pollo CF; Redação - Revisão \& Edição: Meneguin S e Miot HA; Supervisão: Meneguin S. 


\section{REFERÊNCIAS}

1. Brianezi G, Handel AC, Schmitt JV, Miot LD, Miot HA. Changes in nuclear morphology and chromatin texture of basal keratinocytes in melasma. J Eur Acad Dermatol Venereol. 2015;29:809-12. https://doi.org/10.1111/jdv.12453.

2. Tamega AA, Miot LBD, Bonfiete C, Gige TC, Marques ME, Miot HA. Clinical patterns and epidemiological characteristics of facial melasma in Brazilian women. J Eur Acad Dermatol Venereol. 2013;27:151-6. https://doi.org/10.1111/j.14683083.2011.04430.x.

3. Handel AC, Lima PB, Tonolli VM, Miot LD, Miot HA. Risk factors for facial melasma in women: a case-control study. Br J Dermatol. 2014;171:588-94. https://doi.org/10.1111/ bjd.13059.

4. Sociedade Brasileira de Dermatologia. Perfil nosológico das consultas dermatológicas no Brasil. An Bras Dermatol. 2006; 81:549-58. https://doi.org/10.1590/S0365-05962006000600005.

5. Corrêa MP, Pires LCM. Doses of erythemal ultraviolet radiation observed in Brazil. Int J Dermatol. 2013;52(8):96673. https://doi.org/10.1111/j.1365-4632.2012.05834.x.

6. CestariTF, Hexsel D, Viegas ML, AlmeidaART, Mendes AMD, Filho JWA, et al. Validation of a melasma quality of life questionnaire for Brazilian Portuguese language: the MelasQoL-BP study and improvement of QoL of melasma patients after triple combination therapy. $\mathrm{Br} J$ Dermatol. 2006;156(1):13-20. https://doi.org/10.1111/j.1365-2133.2006.07591.x.

7. The World Health Organization Quality of Life Assessment (WHOQOL): position paper from the World Health Organization. Soc Sci Med. 1995;41:1403-9.

8. Romero M, Vivas-Consuelo D, Alves-Guzman N. Is Health Related Quality of Life (HRQOL) a valid indicator for health systems evaluation? Springerplus. 2013;2(1):664-70. https:// doi.org/10.1186/2193-1801-2-664.

9. Taborda MLW, Weber MB, Freitas ES. Avaliação da prevalência de sofrimento psiquico em pacientes com dermatoses do espectro dos transtornos psicocutâneos. An Bras Dermatol. 2005;80:351-4. https://doi.org/10.1590/ S0365-05962005000400004

10. Padilha S, Noronha APP, Fagan CZ. Instruments for psychological assessment: use and referee by psychologists. Aval. psicol. 2007;6(1):69-76.

11. Beck CLC, Gonzales RMB, Leopardi MT. Técnicas e procedimentos de pesquisa qualitativa. In: Leopardi MT. Metodologia da pesquisa na saúde. Santa Maria: Palotti; 2001. p. 223-44.

12. Dall'Agnol CM, Trench MH. Grupos focais como estratégia metodológica em pesquisas na enfermagem. R. gaúcha Enferm. 1999;20(1):5-25.

13. Bardin L. Análise de conteúdo. Lisboa: Edições 70; 2009.
14. Ludwig MWB, Muller MC, Oliveira MS, Moraes JFD. Qualidade de vida e localização da lesão em pacientes dermatológicos. An Bras Dermatol. 2009;84(2):143-50. https://doi.org/10.1590/S0365-05962009000200007.

15. Jesus PBR, dos Santos I, Brandão ES. La autoimagen de símismo y la autoestima de las personas com transtornos de piel: uma revisión integradora de la literatura la luz de la teórica Callista Roy. Arquichan. 2015;15(1):75-89. https:// doi.org/10.5294/aqui.2015.15.1.8.

16. Alves GF, Nogueira LSC, Varella TCN. Dermatologia e gestação. An Bras Dermatol. 2005;80(2):179-86.

17. Taylor A, Pawaskar M, Taylor SL, Balkrishnan R, Feldman SR. Prevalence of pigmentary disorders and their impact on quality of life: a prospective cohort study. J Cosmet Dermatol. 2008;7(3):164-8. https://doi.org/10.1111/j.14732165.2008.00384.X.

18. Scherdin U, Burger A, Bielfeldt S, Filbry A, Weber T, Scholermann $A$, et al. Skin-lightening effects of a new face care product in patients with melasma. J Cosmet Dermatol. 2008;7(1):68-75. https://doi.org/10.1111/j.14732165.2008.00364.x.

19. Finlay AY, Khan GK. Dermatology Life Quality Index (DLQI): a simple practical measure for routine clinical use. Clin Exp Dermatol. 1994;19(3):210-6.

20. Abdel-Hafez K, Mahran AM, Hofny ER, Mohammed KA, Darweesh AM, Aal AA. The impact of acne vulgaris on the quality of life and psychologic status in patients from upper Egypt. Int J Dermatol. 2009;48:280-5. https://doi.org/10.1111/ j.1365-4632.2009.03838.x.

21. Ozelame DM. Pensamento complexo, educação e aprendizagem. Revista Espaço Acadêmico. 2015;15(170):80-6.

22. Tasoula E, Gregorius S, Chalikias J, Lazarou D, Kanopoulou I, Katsambas A, et al. The impact of acne vulgaris on quality of life and psychic health in young adolescents in Greece. Results of a population survey. An Bras Dermatol. 2012;87:862-9. https://doi.org/10.1590\%2FS0365-05962012000600007.

23. Miot LBD, Miot HA, Silva MG, Marques MEA. Fisiopatologia do melasma. An Bras Dermatol. 2009;84(6):623-35. https:// doi.org/10.1590/50365-05962009000600008.

24. Belletti MUM. Conhecimento, atitude e prática da equipe de saúde sobre melasma na gravidez. Av Enferm. 2018;36(1):409. https://doi.org/10.15446/av.enferm.v36n1.58896.

25. Parada M, Teixeira SP. Maquiagem e camuflagem. São Paulo: Moreira Jr; 2010.

26. Jiang J, Akinsseye O, Tovar-Garza A, Pandya AG. The effect of melasma on self-esteem: a pilot study. Int J Women Dermatol. 2018;4(1):38-42. https://doi.org/10.1016\%2Fj. jiwd.2017.11.003. 